\section{Implementation of Problem- Based Learning Method of Medical Education to Improve Trends in Undergraduate Medical Research in Pakistan}

Sir,

Research is cornerstone of discovery and innovation in the field of medicine. Scientists created effective treatments for diseases once thought life-threatening and incurable, with the help of medical research on underlying pathophysiological processes. The revolutionary newer direct-acting antiviral treatment for hepatitis is a meritorious success of clinical research. In the current times, the management of diseases is highly dependent on evolving medical literature. Therefore, quality research carries paramount importance in order to excel in the field of medicine. It fosters critical thinking, understanding of literature, decision-making and sound clinical-knowledge base, which are the key skills in clinical practice. Unfortunately, South Asia contributes only $1.2 \%$ of the research projects annually. ${ }^{1}$ Considering the ever-changing disease patterns, emerging new infectious and non-infectious diseases, and sky-high under-five mortality rate (78.8/1000 live births) in Pakistan, it is immensely important for the medical students to direct their efforts towards research. ${ }^{2}$

The research culture in Pakistan is still in the initial stages and we are quite lagging behind. It is a matter of particular urgency for health-policy makers to promote research trends among undergraduate medical students in order to improve the prevention as well as treatment outcomes by maintaining a reliable healthcare system. The major problems that hinder the process of medical research in Pakistan are inadequate research and development budget; lack of interest, time, and opportunities; lack of research curriculum; and most importantly the conventional method of medical education in majority of medical institution s in Pakistan. ${ }^{1}$

Compared to the conventional lecture-based learning $(\mathrm{LBL})$, problem-based learning ( $\mathrm{PBL}$ ) is a studentcentered pedagogy. It promotes practicality, emphasizes on enhancing knowledge, and instils critical thinking in undergraduate medical students. A better understanding and knowledge of diseases, as reflected by relatively better academic performance of students through PBL as opposed to LBL $(p=0.001)$, can help to conduct quality research studies. ${ }^{3}$ Haseeb et al. highlighted that the PBL group of students showed healthier attitudes and better knowledge towards health research $(p<0.001)$, as compared to their LBL counterparts. ${ }^{4}$ Khan et al. endorsed that the PBL students had a researchoriented attitude when compared with the LBL students $(p=0.021)$, and they were more inclined to actively participate in research $(p=0.017) .5$

Medical students, taught in traditional approach, place more emphasis on the resources supplied by the faculty; in contrast, PBL-trained students have an approach that empowers reasoning and conducting research in order to solve the problems and improve the learning. ${ }^{5}$ According to an audit report, major chunk of all articles published in the students' corner of the Journal of Pakistan Medical Association originated from medical colleges where method of medical education was PBL. 6 In Pakistan, a study highlighted that postgraduate trainees were deficient practically in terms of reading and writing medical literature. ${ }^{7}$ As PBL showed marked improvement in clinical reasoning of medical students as compared to traditional methods, it can also help to facilitate the development of the local literature both in terms of research utilisation and production.

A study showed significant improvement in the students' problem solving and critical thinking skills $(p<0.001)$, establishing the fact that students who undergo PBL training are more likely to have better learning and professionally pertinent skills after graduation. They are more actively engaged in analyses and applications necessary for clinical trials; hence, more inclined towards research. ${ }^{8} \mathrm{PBL}$ is a means of promoting self-learning, enhancing communication skills and problem solving capacity. The result of a study demonstrated that introduction of PBL method significantly motivated students to identify the gaps in their knowledge and stimulated $76 \%$ students to conduct research. ${ }^{9}$ Hence, the research-friendly impact of the PBL curricula may help in improving research output in Pakistan.

The deteriorating healthcare in Pakistan is an immense burden on our economy. It warrants meticulous research pertaining to regional public health issues in Pakistan that will not only pinpoint the leading healthcare delivery problems but will also help to develop cost-effective clinical practice, and ultimately improving the quality of life of the individuals. Poor research quality and low research output among postgraduates reflect a lack of research skill and implementation of research-oriented curriculum during their undergraduate years. Therefore, developing the curricula in terms of PBL may help to develop an insight of the research methodology, inculcate critical thinking, sharpen reasoning skills, and enhance productive approaches in medical students. It will improve the quality of undergraduate research that 
can partly compensate for the overall lack of research in medical profession in Pakistan. Hence, it should be greatly emphasised to adopt PBL curricula, in order to develop an optimistic attitude towards medical research. Health policy-makers should give a trial to $\mathrm{PBL}$ implementation in medical colleges in Pakistan and the data should be collected regarding changes in the research outcomes.

\section{REFERENCES}

1. Fatima M, Zehra N, Ahmad F, Obaid MS. Awareness regarding research skills among clinical and academic post graduate doctors in teaching hospitals of Karachi. J Pak Med Assoc 2014; 64:624-8.

2. Pak-UNICEF Data 2016. https://data.unicef.org/country/pak/ Accessed: April 29, 2018.

3. Faisal R, Bahadur S, Shinwari L. Problem-based learning in comparison with lecture-based learning among medical students. J Pak Med Assoc 2016; 66:650-3.

4. Haseeb A, Bilal M, Ansari MA, Raheem A, Khan A, Arshad MH, et al. Impact of mode of curriculum on knowledge and attitudes of medical students towards heath research. J Clin Diagn Res 2016; 10:LC15-9.

5. Khan H, Taqui AM, Khawaja MR, Fatmi Z. Problem-based versus conventional curricula: influence on knowledge and attitudes of medical students towards health research. PLOS One 2007; 2:e632.

6. Aslam F, Waheed A. An audit of the students' corner of Journal of the Pakistan Medical Association. J Pak Med Assoc 2005; 55:517-9.

7. Aslam F, Qayyum MA, Mahmud H, Qasim R, Haque IU. Attitudes and practices of postgraduate medical trainees towards research--a snapshot from Faisalabad. J Pak Med Assoc 2004; 54:534-6.

8. Tayyeb R. Effectiveness of problem based learning as an instructional tool for acquisition of content knowledge and promotion of critical thinking among medical students. J Coll Physicians Surg Pak 2013; 23:42-6.

9. Habib F, Baig L, Mansuri FA. Opinion of medical students regarding problem-based learning. J Pak Med Assoc 2006; 56:430-2.

Maha Jahangir ${ }^{1}$ and Faisal Inayat ${ }^{2}$

1 Dow Medical College, Karachi, Pakistan

2 Allama Iqbal Medical College, Lahore, Pakistan

Correspondence: Dr. Faisal Inayat, Department of Medicine, Allama Iqbal Medical College, Allama Shabbir Ahmad

Usmani Road, Faisal Town, Lahore-54550, Pakistan

E-mail:faisalinayat@hotmail.com

Received: April 30, 2018; Accepted: June 29, 2018 .......... 